psychopraxis. neuropraxis $2021 \cdot 24: 62-69$ https://doi.org/10.1007/s00739-020-00681-1 Online publiziert: 11. November 2020 (c) Springer-Verlag GmbH Austria, ein Teil von Springer Nature 2020

Obgleich alternative Settings wie etwa videogestützte Therapien keine Neuheit darstellen, führte der COVID-19Ausbruch zu einem exponentiellen Anstieg dieses Therapieformats-, a turning point for e-health" - und es ist davon auszugehen, dass dieses „neue“ Format der Therapie-Community zumindest anteilig erhalten bleibt. Auch die Autismustherapie erfindet sich aktuell insofern neu, als überdurchschnittlich viele Therapiesitzungen im Videoformat abgehalten werden. Neben den Herausforderungen dieses Umdenkprozesses und den zu bewerkstelligenden Anpassungsleistungen aller Beteiligten offenbaren sich - nicht nur angesichts neu gesammelter Erfahrungen - die Implikation und Erfolgschancen dieses Formats, das im hiesigen Kontext als Ergänzung und nicht als Ersatz für klassische Therapieformate $\mathrm{zu}$ verstehen ist.

\section{Videogestützte Autismus- therapie: ein Neuland?}

Vorerst ist aufgrund der Vielzahl an Bezeichnungen eine Begriffsbestimmung indiziert. In der Literatur lassen sich nomenklatorisch vor allem Begriffe wie Online-Therapie, Online-Beratung, Online-Intervention, internetbasierte Therapie und E-Mental-Health finden, die als übergeordnete Bezeichnungen $\mathrm{zu}$ verstehen sind und teilweise - je nach Ansatz und Konzept - videogestützte Therapien einschließen. Der Fokus im vorliegenden Beitrag soll auf der videogestützten Therapie liegen, das heißt auf therapeutischen Kontakten über das

Literatur beim Verfasser

\title{
Anas Nashef
}

Autismus-Therapiezentrum, Bremerhaven, Deutschland

\section{Videogestützte Autismustherapie: Implikation, Gestaltung und Wirksamkeit}

Medium Videotelefonie zwischen Behandlern und Behandelten, zwischen Therapeuten und Klienten. Hierbei werden die Kontakte in der Regel nicht ausschließlich über Video abgehalten, sondern auch vis-à-vis und vereinzelt über Telefonie und E-Mails, und sie umfassen darüber hinaus beratende Tätigkeiten mit dem Umfeld des Klienten.

Bereits seit 2019 (vor der COVID19-Krise) können niedergelassene Psychotherapeuten in Deutschland Videosprechstunden anbieten. Die Anfänge der Online-Beratung liegen nach Eichenberg und Kühne in der zweiten Hälfte der 1990er-Jahre und E-MentalHealth besteht seit etwa 20 Jahren. Es folgten Angebote $\mathrm{zu}$ Online-Therapien, die störungsspezifisch sind und oft die Behandlung affektiver Störungen zum Ziel hatten. Bereits 2000 wurde der Begriff E-Health definiert und im psychologischen Kontext als „behavioral eHealth" bezeichnet, womit psychologische Interventionen gemeint sind. Im Unterschied zur Beratung wird OnlineTherapie charakterisiert durch längere Kontakte, welche auf die Behebung von klinisch relevanten Störungen abzielen. Online-Angebote beinhalten nicht notwendigerweise eine videogestützte therapeutische Arbeit, sondern bedienen sich anderer Modi wie OnlineManuals mit oder gar ohne direkten therapeutischen Kontakt.

Während die Internetsuche nach videogestützten Therapien Tausende Treffer ergibt, bleiben die Ergebnisse bei der Aufnahme eines Autismusbezugs überschaubar. Hier beziehen sich die Treffer vor allem auf den Marte-Meo-Ansatz, der vis-à-vis videogestützt operiert, indem
Videos mit unterschiedlichen Interaktionspersonen nach bestimmten Kriterien einer beziehungsorientierten Analyse gemeinsam etwa mit den Bezugspersonen unterzogen werden. Eine ähnliche Arbeit mit behavioralem Hintergrund vor allem im Sinne eines „video modelling $[s]$ “ sowie eine Online-Beratung mit autismusspezifischem verhaltenstherapeutischem (AVT)-Background werden ebenfalls angeboten (vgl. BernardOpitz $)^{1}$.

\section{Vorüberlegungen und Vorbereitung der video- gestützten Autismustherapie}

Im Unterschied zum „video modelling“ oder zu internetbasierten Therapieangeboten ohne einen direkten TherapeutenKlienten-Kontakt wird hier eine videogestützte Autismustherapie beschrieben, die stets im Kontakt - vis-à-vis und videogestützt - erfolgt und durch Schreibkontakte via E-Mail oder Chat oder durch Telefonie ergänzt werden kann. Ebenso wie die klassische Vis-à-visTherapiearbeit zielt diese Intervention auf Milderung von mit Autismus-Spektrum-Störungen (ASS) einhergehenden Interaktionsproblemen, Brückenbauen zwischen autistischen und nichtautistischen Perzeptionen im sozialen Raum, Stärkung der Mentalisierungsfähigkeiten und insgesamt die Ermöglichung der gesellschaftlichen Teilhabe von betroffenen Kindern, Jugendlichen und Erwachsenen mit ASS ab.

\footnotetext{
1 http://www.verabernard.de/onlineberatung. htm [Zugriffam 21.07.2020].
} 
Hier steht eine Anzeige.

\section{曾 Springer}


In einem ersten Schritt ist es von Relevanz, dass eine Entscheidung für einen Videokonferenzanbieter getroffen wird. Auf dem Markt existieren diverse Anbieter ${ }^{2}$, welche Videoprogramme nicht ausschließlich für den therapeutischen Kontext - bereitstellen. Bei der Wahl eines Anbieters sind zuvörderst folgende Faktoren zu berücksichtigen:

1. Ist der Anbieter zertifiziert?

2. Wie ist es mit dem Datenschutz bestellt?

3. Ist die Nutzung kostenpflichtig und wenn ja: Welche Kosten entstehen?

4. Ist die Plattform nur für zwei Personen zugelassen oder können auch Gruppensettings durchgeführt werden?

Im Vorfeld der videogestützten Therapie ist es zudem erforderlich, den Rahmen mit den Klienten und den Sorgeberechtigten zu besprechen und ihre schriftliche Einwilligung einzuholen. Hierbei müssen vor allem folgende Punkte Beachtung finden: die Einwilligung zur Nutzung dieses Formats, die Vertraulichkeit der Sitzungen und das beidseitige Verbot von Ton- und Bildaufnahmen sowie die jederzeitige Möglichkeit der Rücknahme der Einwilligung. Darüber hinaus ist es besonders indiziert, die technischen Voraussetzungen und die Regeln des Videoformats im Vorfeld zu besprechen und diese allenfalls zu verschriftlichen. $\mathrm{Zu}$ diesen Regeln gehört, dass sich keine anderen Personen ohne Wissen der Beteiligten im geschützten therapeutischen Raum (Räumen) aufhalten dürfen, dass Störungen zu minimieren bzw. zu verhindern sind, dass andere Kommunikationsmittel wie etwa Mobiltelefone auszuschalten sind, dass auf die Lichtverhältnisse geachtet wird und dass Gesicht- und Oberkörper zu sehen sind.

Insbesondere im Kontext der Autismustherapie müssen darüber hinaus weitere Faktoren beachtet werden. So herrscht Einigkeit darüber, dass Men-

\footnotetext{
2 Eine Liste zertifizierter Videodienstanbieter wird von der Kassenärztlichen Bundesvereinigung (KBV) zur Verfügung gestellt: https://www.kbv.de/media/sp/Liste zertifizierte_Videodienstanbieter.pdf [Zugriff am 21.07.2020].
}

schen mit Autismus durch Außenreize schnell „überflutet“"werden können. Dies kann zu Unruhe und zu erhöhtem Arousal führen und lenkt ab bzw. führt $\mathrm{zu}$ Irritationen bei dieser Klientengruppe, sodass das Setting präventiv so zu gestalten ist, dass sowohl auf Therapeutenals auch auf Klientenseite sensorische und andere Störungen ausbleiben. Indem beispielsweise ruhige Orte gewählt werden und die jeweilige Sitzung mit geschlossenen Fenstern und Türen abgehalten wird, können Nebengeräusche reduziert werden. Auch empfiehlt sich, den Hintergrund so zu wählen, dass dieser möglichst reizarm ist. Bei einigen Anbietern kann zudem während der Videositzung das eigene Bild gesehen werden, ein Umstand, der ebenfalls bisweilen ablenkend wirkt und dementsprechend - wenn technisch möglich zu vermeiden ist.

Des Weiteren ist die Ausrichtung der Webcam von Relevanz, um die Sichtbarkeit von Mimik und Gestik zu gewährleisten. Insgesamt offenbart sich syndromimmanent das maximal mögliche Zur-Verfügung-Stellen relevanter kommunikationsbezogener Informationen als zentral. Angesichts der klassifikatorischen Schwierigkeit der Deutung nonverbaler Signale bei Menschen mit Autismus erscheint eine verbale Kompensation während des Videosettings unverzichtbar. Deshalb empfiehlt es sich, ein ökonomisches Fragesystem zu nutzen, um Rückmeldungen des Klienten als wiederholten Baustein innerhalb der jeweiligen Sitzung zu gewährleisten. Ein weiterer Aspekt bezieht sich auf die Blickkontaktaufnahme: Ein Blickkontakt wie im analogen Gespräch ist nicht gegeben, da die Gesprächspartner in der Regel nicht in die Kamera, sondern auf den Bildschirm schauen. Dadurch kann der Blickkontakt jedoch starr und fixiert wirken, was Menschen mit Autismus als besonders störend empfinden können. Als hilfreich erwies sich, wenn der Therapeut/die Therapeutin gelegentlich bewusst den eigenen Blickkontakt „bewegt“. Während der Interaktion zeigen sich ebenfalls bisweilen - vor allem bei Kindern - Schwierigkeiten im Erkennen der Gesprächsstruktur, sodass die Einführung von gestischen Signalen, um etwa auf das Ende eines Gesprächsbeitrages hinzuweisen, bei manchen Klienten indiziert erscheint. Im gesamten Ablauf der jeweiligen Sitzung können zudem sogenannte TEACCH-Leisten eingesetzt werden, welche die Stundenstruktur durch Fotos bzw. Piktogramme visuell wiedergeben und die jeweilige Stunde entsprechend vorhersehbarer machen.

\section{Behandlungsrelevante Themen und Aspekte}

Im Folgenden werden behandlungsrelevante Themen und Aspekte skizziert, welche der gewöhnlichen Praxis der Autismustherapie entspringen und eine besondere Relevanz im Kontext der Videoarbeit besitzen bzw. eine besondere Beachtung verdienen. Hierbei handelt es sich nicht um einen einzigen theoretischen Hintergrund, sondern um unterschiedliche Ansätze.

\section{Beginn und Ende der Sitzung}

Vorausgeschickt lässt sich formulieren, dass das Videosetting per se strukturfördernd ist: Neben dem deutlichen Rahmen der Bildschirme als Ort des therapeutischen Geschehens sind der Beginn und das Ende der Sitzung visuell klar umrissen: der Beginn mit Erscheinen der Beteiligten auf dem Bildschirm und das Ende mit deren Verschwinden vom Bildschirm. Wie in den Vis-à-vis-Sitzungen auch profitieren Klienten mit ASS - wie das TEACCH-Modell insgesamt postuliert - von einem umrissenen und ritualisierten inhaltlichen Anfang und Ende der jeweiligen Sitzung und ebenfalls dessen strukturierten und als solche kenntlich gemachten Bausteinen. Sowohl als Einstieg in die Sitzung als auch vor deren Abschluss können individualisiert visualisierte Gefühlsskalen eingesetzt werden. Freilich im Rahmen einer Videotherapie, in der Hinweissignale wie Gestik eingeschränkt wahrnehmbar sein können, und insgesamt in Anbetracht syndromimmanent mangelnder Kommunikation offenbart sich die explizite therapeutenseitige Nachfrage einer Rückmeldung zur therapeutischen Sitzung als wichtiges Therapieelement, das ebenfalls in Skalenform angeboten werden kann. 
Umgang mit dem fehlenden Spiel als wichtiges Medium in der Kindertherapie

Gerade im Kindesalter stellt das Spiel bekanntlich ein zentrales therapeutisches Medium dar, in dessen Rahmen in der Sprache des Kindes interagiert und auch gehandelt und „korrigiert“ bzw. contained und modifiziert werden kann. Das Spiel dient nicht nur der zeitlichen Ritualisierung therapeutischer Sitzungen, indem es etwa als Abschlussritual eingesetzt wird. Vielmehr geht es hierbei um ein entwicklungspsychologisch sehr zentrales und wertvolles therapeutisches Mittel, das die kindliche Reflexion alltäglicher Interaktionen, Emotionen und Konflikte ermöglicht. So werden Situationen und Konflikte spielend zum Ausdruck gebracht, Perspektiven erlebt, Regeln erlernt und Lösungen entwickelt. Daher stellt sich die Frage, mit welchen Alternativmitteln im Videokontakt bei Kindern Emotionen, Konflikte, Differenzen, Perspektiven spielerisch und kindgerecht Berücksichtigung finden können. Hier können Onlinespiele zumindest einen Teilersatz für diese direkten Spiele im und außerhalb des therapeutischen Raumes darstellen. In Anbetracht der Motive des Computerspiels für Kinder und Jugendliche offenbart sich deutlich, dass diese auch Gegenstand therapeutischer Interventionen sind.

\section{) Gesellschafts- und \\ Kooperationsspiele als Kontaktangebot für Kinder}

Im Kontext der Autismustherapie kann der videogestützte Kontakt beziehungsaufbauend dafür genutzt werden, Lieblingsobjekte und Spezialinteressen der Kinder direkt gezeigt zu bekommen. Diese sind vor allem in der Autismustherapie von immenser Bedeutung und stellen einen wichtigen therapeutischen Motivations- und Wirkfaktor dar. Auch können verschiedene Gesellschafts- und Kooperationsspiele während des Videochats gespielt werden, die sich als Kontaktangebot mit anderen Kindern und Jugendlichen außerhalb des therapeutischen Settings installieren lassen

psychopraxis. neuropraxis 2021 · 24:62-69 https://doi.org/10.1007/s00739-020-00681-1

(c) Springer-Verlag GmbH Austria, ein Teil von Springer Nature 2020

\section{Videogestützte Autismustherapie: Implikation, Gestaltung und Wirksamkeit}

\section{Zusammenfassung}

Obgleich alternative Settings, wie etwa videogestützte Therapien, keine Neuheit darstellen, führte der COVID-19-Ausbruch zu einer explosiven Zunahme dieses Therapieformats und es ist davon auszugehen, dass dieses „neue" Format der Therapie-Community zumindest anteilig erhalten bleibt. Auch in der Autismustherapie werden aktuell überdurchschnittlich viele Therapiesitzungen im Videoformat abgehalten. Neben den Herausforderungen dieses Umdenkprozesses und der zu bewerkstelligenden Anpassungsleistung aller Beteiligten offenbaren sich nicht nur angesichts neu gesammelter Erfah- rungen - die Implikation und Erfolgschancen dieses Formats deutlich, das im hiesigen Kontext als Ergänzung zu verstehen ist. Dieser Beitrag möchte in die videogestützte Autismustherapie einführen, mögliche therapeutische Interventionen skizzieren und sich mit dem Forschungsstand hinsichtlich der Wirksamkeit dieser Therapieform befassen.

Schlüsselwörter

Autismus-Spektrum-Störungen (ASS) · Intervention · Mentalisierung · Videotherapie . E-Health

\section{Video-Supported Autism Therapy: Implication, Design and Efficacy}

\section{Abstract}

Although alternative settings like videosupported therapies are nothing new, the outbreak of COVID-19 has led to an explosive increase in this type of therapy and it is expected that this "novel" format of the therapeutic community will at least retain its proportionate share. At present, autism therapy also holds an above-average number of therapy sessions using the video format. Apart from the challenges of this rethinking process and the need for all participants to adapt, the implications and chances of success of this format, which should be understood to be supplementary in the local context, are becoming manifest, not only in view of the newly gained experience. This article is aimed at introducing videosupported autism therapy, outlining possible therapeutic interventions, and discussing the state of research in terms of the efficacy of this form of therapy.

\section{Keywords}

Autism Spectrum Disorders (ASD) · Intervention - Mentalization - Video therapy · E-Health
(z.B. Minecraft als Servermöglichkeit). Alternativ zu Online- und zu Vis-à-visSpielen können verschiedene verbale und mentalisierungsfördernde Spiele während des Videochats eingesetzt werden (s. unten); eine Reihe solcher "Spiele“ und Aktivitäten für das Einzel- und Gruppensetting können der Multifamilientherapie entnommen werden.

Klassische Sprach- und Wortspiele eignen sich ebenfalls für die Videokontakte so etwa „Teekesselchen“, „Stadt, Land, Fluss“, „Wer bin ich?“ oder „Ich packe meinen Koffer". Diese Spiele können zudem so variiert werden, dass sie aktuelle therapeutische Themen zum Gegenstand haben. So lässt sich der Kof- fer im Spiel „Ich packe meinen Koffer“ mit themenspezifischen Inhalten füllen, etwa mit Eigenschaften oder Gefühlen, die mitgenommen werden sollten, oder mit Mutmachern etc. Ebenso kann das Spiel „Wer bin ich?" mit einer umrissenen Menschengruppe gespielt werden, etwa mit Menschen, mit denen die Spieler viele Eigenschaften oder keine Eigenschaften teilen, mit Idolen, mit Menschen aus einer bestimmten Berufsgruppe usw. Eine weitere Spielmöglichkeit bieten gängige Gesellschaftsspiele, die sowohl im Therapeutenzimmer als auch beim Kind zu Hause vorhanden sind. 


\section{Der Klientenraum als Interaktions-} grundlage

Als Besonderheit und zugleich als Chance erweist sich die Videotherapie, während der sich der Klient meist im eigenen Heim oder im eigenen Zimmer aufhält. Neben dem Umstand, dass beispielsweise das eigene häusliche Zimmer einen in besonderem Maße sicheren und vertrauten Ort verkörpert, befindet sich der Klient in der Regel in nächster Nähe zu ihm wichtigen „autistischen“ Objekten, welche im Sinne der Klassifikationssysteme ein Spezialinteresse darstellen können oder schlichtweg wichtig für den Klienten sind. Wie bereits an einer anderen Stelle ausgeführt, können diese Objekte utilisierend im Rahmen der Intervention - oder besser Interaktion - im Sinne einer Deobjektivierung und affektiven Erweiterung dieser Objekte genutzt werden. Neben dem Klientenraum im eigentlichen Sinne bietet sich ferner die Möglichkeit an (bei der klientenseitigen Nutzung eines Mobiltelefons), dass der Klient seinen „Lebensraum“ via Videofunktion vorstellt, wie z. B. das eigene Zuhause bzw. Orte zu Hause, an denen sich der Klient gern aufhält, oder die nahe Umgebung oder der Stadtteil.

\section{Entdeckung des Affekts, des} anderen und des Affekts des anderen

Aus der Autismusforschung stammt das Konzept der „Theory of Mind (ToM)“, welches die Fähigkeit des Zuschreibens mentaler Zustände bei anderen beschreibt. Autismus-Spektrum-Störungen werden hierbei mit einer Schwäche dieser Fähigkeit assoziiert, wobei nach dieser Leseart - in Anbetracht der starken Verbindung der sogenannten „False-Belief-Aufgaben“ mit dem Theoryof-Mind-Konzept - die Fähigkeit zur Perspektivübernahme zentral ist. Das Videosetting, in dem sich der Klient in seinem häuslichen Umfeld und der Therapeut im Therapieinstitut befindet, bietet einen Rahmen, in dem sich eine Sensibilisierung für diese unterschiedlichen Perspektiven sogar leichter als im Vis-à-Vis-Setting operationalisieren lässt. So unterscheiden sich viele per- spektivenbezogene Aspekte wie etwa die sensorischen Eindrücke, die eine gemeinsame Grundlage eines therapeutischen Erforschens bieten können.

\section{》) Mentalisierungsansatz als ToM-Weiterentwicklung}

Gewissermaßen eine Weiterentwicklung des kognitionspsychologischen ToMKonzepts verkörpert der Mentalisierungsansatz, der sich aufgrund der Zentralität seiner verbalen Interaktion ebenfalls für ein Videosetting eignet. Es ist in Anbetracht der ToM-Einschränkungen bei Menschen mit Autismus durchaus frappierend, dass dieser Ansatz bisher nicht genügend Platz in der Autismusforschung und -praxis gefunden hat. Denkbar ist, dass dies auf den kognitiven und behavioralen Fokus bei Autismus in den letzten Dekaden sowie auf die eingebürgerte und transgenerational weitergegebene, zum Teil auch berechtigte Distanzhaltung zu analytisch orientierten und vor allem mit dem Namen Bettelheim assoziierten Arbeiten der sechziger Jahre des letzten Jahrhunderts zurückzuführen ist. Dennoch stellt die Arbeit von Reul et al. eine in jüngster Zeit vielversprechend erprobte Pilotarbeit der mentalisierungsbasierten Therapie im ASS-Kontext dar, die im Gruppensetting mit Erwachsenen mit hochfunktionalen Autismusformen erfolgt.

Ziel der mentalisierungsbasierten Interaktionen ist - auch im Autismustherapiekontext - die Mentalisierung kontextgebunden und im Hier und Jetzt zu fördern, vorherrschenden mentalisierungshemmenden Modi mit entsprechender Technik zu begegnen und die Mentalisierungsfähigkeit entlang der Pole selbst vs. andere, affektiv vs. kognitiv, innen vs. außen und implizit vs. explizit - vor allem durch das sogenannte "contrary move“ - auszubalancieren. In Anbetracht der syndromimmanenten Struktur-, Denkund sozioaffektiven Besonderheiten bieten sich bei Menschen mit ASS - auch im Videosetting - vor allem folgende therapeutische Vorgehensweisen an:

1. Eine neugierige therapeutische Haltung einnehmen, die den Weg zu einer gemeinsamen affektiven Erforschung ebnet.

2. Therapeutische Aufmerksamkeit auf etwaiges Pseudo- und Hypermentalisieren. Neben dem Nichtmentalisieren können bei Menschen mit vor allem hochfunktionaler ASS spezifische pseudomentalisierende Reaktionen betrachtet werden, die sich etwa in Intellektualisierung und Kognitivierung von sozioemotionalen Interaktionen zeigen. Ebenfalls wie bisweilen bei der Beantwortung der Fragen im Videotest MASC anhand von Filmszenen sichtbar ist, kann Hypermentalisieren bei unserer Zielgruppe festgestellt werden. Hypermentalisieren bezeichnet die Tendenz, in sich selbst und den anderen zu viel von Affekten und Motiven "hineinzulesen“.

3. Aufgrund der Tendenz hin zum Kognitiven empfiehlt sich, mit der Technik der contrary moves Affekte $\mathrm{zu}$ fokussieren.

4. Ausgehend von einem vorherrschenden - aber gewiss nicht ausschließlichen und starr zu kategorisierenden Äquivalenzmodus bei Menschen mit ASS soll der Fokus der Interaktion nicht auf das Argumentieren gelegt werden, das in der Regel zu einer wenig psychisch anregenden Argumentieren-GegenargumentierenDynamik und somit zu einer Frontenbildung und Frontenverhärtung führt. Vielmehr empfiehlt sich, neben der o.g. neugierigen Haltung empathisch validierend $\mathrm{zu}$ interagieren und ggf. eigene therapeutenseitige Erfahrungen einzubeziehen sowie eigene Irritationen oder besser Überraschung zu benennen.

Während die genannten Techniken bei Jugendlichen und Erwachsenen im Videosetting ohne Weiteres umsetzbar sind, stellt sich eine ähnliche Vorgehensweise bei Kindern angesichts des verbalen Charakters der oben vorgetragenen Interaktionen sowie der zentralen Rolle des Spiels in der Kindertherapie als zumindest erschwert dar. Anstelle einer Interaktion mit gesprächsintensiven Anteilen können daher mentalisierungsfördernde Medien und Aktivitäten wie Bildkarten, 
Bücher, Spielfiguren sowie Übungen bzw. verbal vermittelte Spiele (s. oben) herangezogen werden. Der Förderungsprozess des Mentalisierens kann sich hierbei an dem Hausbaumodell nach Diez Grieser und Müller orientieren, beginnend mit dem Keller als Basis (Aufmerksamkeitsregulation) über das Parterre (Affektregulation) und den ersten Stock (Mentalisieren im engeren Sinne) bis hin zum zweiten Stock (Selbst- und Beziehungsreflexion).

\section{Von Sitzung zu Sitzung}

Ob videogestützt oder nicht, ist die Bedeutung der Zeit zwischen den Therapiesitzungen besonders hervorzuheben: nicht nur weil die Interaktionen in dieser Zeit im realen und nicht therapeutisch geschützten Raum vonstattengehen, vielmehr bietet die reale Welt einen natürlichen Ort des Erlebens von Affektregungen, Interaktionen, Konflikten, inter- und intrapersonellen Krisen und deren subjektives Perzipieren, Mentalisieren und Regulieren.

Dieser Raum, der - anders als der therapeutische Raum - weder vorhersehbar noch kontrollierbar ist, hat einen besonderen therapeutischen Nutzen. Klassisch könnten hier therapeutische Aufgaben einen Platz finden, die - nicht nur im Autismuskontext - beispielsweise das bewusste Dokumentieren von eigenen
Gefühlsregungen während dieses Zwischenraums umfassen. Doch neben diesen klassischen Aufgaben können onlinebasierte - sogenannte ungeleitete oder Blended-Therapieansätze - ebenfalls genutzt werden, die dann in der Videotherapie zur Sprache kommen und in die therapeutischen Interventionen integriert werden können. Hierbei haben diese Angebote den Vorteil einer täglichen Begleitung und ggf. Rückmeldung, auch wenn sie den persönlichen therapeutischen Kontakt nicht ersetzen, vor allem wenn wir jegliche Intervention als Interaktion begreifen.

Diese oft als Training konzipierten Programme fokussieren oft affektive Erscheinungsbilder wie Depression und fußen theoretisch auf einer kognitiv-behavioralen Grundlage. Novego (http:// www.novego.de) etwa bietet solche kostenpflichtigen - Interventionsprogramme zu verschiedenen psychischen Störungen sowie zur Stressprävention an. Nach Angaben des Anbieters eignet sich Novego als Soforthilfe, etwa um Wartezeiten auf einen Therapieplatz zu überbrücken ${ }^{3}$. Eine noch höhere Verfügbarkeit als virtuelle Alltagsbegleiter weisen Anwendungen in Form von mobilen Apps auf, welche etwa die Er-

3 Weitere Anbieter sind z. B.: www.deprexis.de; www.net-step.de; www.geton-training.de. fassung von Erlebtem und Gefühltem sowie die Durchführung von Übungen in Echtzeit ermöglichen. Eine wissenschaftlich untersuchte Anwendung ist die „moodpath“, eine verhaltenstherapeutisch orientierte Anwendung bei Ängsten und Depressionen, über die regelmäßige Stimmungen und Informationen erhoben sowie Einschätzungen und Behandlungswege zurückgemeldet werden. Syndromimmanent können ebenfalls Programme zum Erkennen fazialer Affekte im Internet übend von Klienten genutzt werden. Ein inzwischen klassisches Emotionserkennungsprogramm ist der „MicromomentaryExpressions-Test “ ${ }^{\star}$.

\section{Videogestützte Gruppentherapie}

Zwar können sich die Zielsetzung und die Technik der Gruppentherapie mit Menschen mit ASS je nach theoretischem Hintergrund unterscheiden, die Wichtigkeit und gar Notwendigkeit gruppentherapeutischer Arbeit mit Menschen mit Autismus bleibt aber als notwendige Ergänzung für die Einzeltherapie unumstritten. So sind im Vergleich mit der Einzeltherapie ungeachtet der zur Anwendung kommenden

\footnotetext{
4 http://www.mifor.de/MME/anleitunMME. htm [Zugriffam 12.08.2020].
}

Hier steht eine Anzeige. 
therapeutischen Methoden andere und gruppenspezifische Wirkfaktoren am Werk wie beispielsweise interpersonales Lernen, Kohäsion, Affektspiegelung, Universalität des (störungsspezifischen) Leidens und Altruismus.

Neben den überwiegend manualisierten, in der Regel verhaltenstherapeutisch orientierten Gruppentherapien für Kinder, Jugendliche oder Erwachsene, deren Fokus das Sozialkompetenztraining ist, hat sich die Multifamilientherapie als Gruppenangebot bewährt, das eine simultane therapeutische Arbeit mit bis zu acht Familien darstellt und auf die Stärkung des Familiensystems und der Ressourcen aller Subgruppen abzielt.

\section{》) Gruppentherapeutische Arbeit als Ergänzung für Einzeltherapie unumstritten}

Obwohl videogestützte Gruppentherapien durchaus in anderen Kontexten eingesetzt werden, scheinen diese im $\mathrm{Au}-$ tismuskontext - fast - Neuland zu sein. Vaimberg und Vaimberg berichten dort von einer Gruppentherapie mit vier Kindern und Jugendlichen mit ASS im Alter von 11-14 Jahren, die teils vis-àvis und teils online stattfindet. Hier findet das Medium Online-Spiel (Minecraft) einen zentralen Platz als motivierende Zugangsmöglichkeit zu den Gefühlsregungen der Teilnehmenden. Unsere ersten Erfahrungen mit Kleingruppen, etwa mit vier Jugendlichen mit ASS, sind ebenfalls ermutigend und zeigen, dass interaktionelle Interventionen auch digital gelingen können.

\section{Elternarbeit}

Dass die Elternarbeit ein integraler Bestandteil der Autismustherapie für Kinder und Jugendliche ist und dass diese ohne Elternarbeit nicht auskommt, ist schulübergreifend unumstritten und wird in einer unlängst erschienenen Längsschnittstudie bestätigt. Um den Rahmen nicht zu überschreiten, wird der Fokus hier ausschließlich auf die videogestützte Elternarbeit gerichtet. Das übergeordnete Ziel hierbei lässt sich so formulieren, dass die Eltern- arbeit in der Kinder- und Jugendpsychotherapie insgesamt die Verbesserung der Beziehungsqualität und der Erziehungskompetenzen ins Auge fasst. Unter Berücksichtigung des autistischen Symptombildes insgesamt und der sich häufig ähnelnden Erfahrungswerte der Eltern im sozialen Kontext (mit den Nachbarn, mit der Schule etc.) sowie der begleitenden Trauer können die verschiedenen Interventionen direkte fokussierte Anleitungen, psychoedukative Inhalte, ressourcenstärkende und systemische Fokusse, mentalisierungsfördernde Interaktionen und vernetzungsfördernde Angebote einschließen. Diese und andere Interventionen und Interaktionen lassen sich problemlos im Rahmen der Videotelefonie realisieren.

Von besonderer Wichtigkeit ist es darüber hinaus, die Eltern sowohl im Vorfeld als auch später regelmäßig über die videogestützte Therapie mit ihrem Kind aufzuklären. Auch Hinweise zu den Settingregeln sind wichtig, z. B. dass die Sitzungen ohne Störungen stattfinden, oder welche Termine für den Jugendlichen, welche für die Eltern und welche gemeinsam sind. Mögliche Sorgen oder Fragen der Eltern auch konkret hinsichtlich der Wirksamkeit der Videotherapie sind hierbei ernst zu nehmen, intensiv zu besprechen und unter Umständen Settingänderungen in Erwägung zu ziehen und $\mathrm{zu}$ implementieren.

\section{Zur Wirksamkeit}

Die Wirksamkeit von virtuellen und videogestützten Therapieangeboten, auch in Gruppen (vgl. Gentry et al.), belegen zahlreiche Studien in unterschiedlichen therapeutischen Kontexten. Obgleich Online-Therapien für Erwachsene besser untersucht sind und viele empirische Ergebnisse vorliegen, lassen sich gute Outcomes ebenfalls bei Online-Therapien im Kindes und Jugendalter feststellen. Es ist durchaus verblüffend, dass der erste Versuch, psychotherapeutisch medial $\mathrm{zu}$ intervenieren, über 40 Jahre zurückliegt. Straker et al. berichteten 1976 von Interventionen per TV-Konferenz mit Kindern und deren Müttern.

Rezente Metaanalysen wie etwa Domhardt et al. belegen die vergleichbar mit
Vis-à-vis-Angeboten gute Wirksamkeit von Online-Interventionen. Vor allem Blended-Interventionen weisen gute Wirksamkeiten auf, wie Wirksamkeitsstudien insbesondere bei Angststörungen zeigen (z. B. Hedman et al.). Metaanalysen von Online-Therapien bei depressiven Störungen zeigen womöglich aufgrund von Unterschieden zwischen geleiteten und ungeleiteten Methoden divergierende Ergebnisse, wobei geleitete Interventionen mittlere bis hohe Effekte zeigen. Insgesamt sind das Vorhandensein und die Intensität des direkten therapeutischen Kontakts maßgeblich für eine höhere Wirksamkeit (ebd.). Basierend auf den Ergebnissen vieler Studien liegt eine gute Wirksamkeit bei Depression, sozialer Phobie und Panikstörung vor (vgl. Schuster et al.). Explizit videogestützte Therapien werden insgesamt weniger erforscht, obgleich diese dem Vis-à-visSetting am ehesten nahekommen. Eine Metaanalyse liefert jedoch ermutigende Ergebnisse und zeigt, dass Videotherapie altersunabhängig zu den gleichen Erfolgsergebnissen wie bei Vis-à-visTherapien kommt. Ebenfalls werden Erfolge in der therapeutischen Arbeit mit Eltern und kleinen Kindern mit psychodynamischen Mitteln präsentiert.

\section{》) Gute Wirksamkeit von Online- Interventionen}

Im ASS-Kontext berichten Pennefather et al. von Erfolgen im Rahmen der Online-Trainings für Eltern mit 4- bis 8-jährigen Kindern mit Methoden der ABA (applied behavior analysis) und ACT (acceptance and commitment training), die sich vor allem in elterlicher Stressreduktion und einer Zunahme kindlichen prosozialen Verhaltens zeigten. Insgesamt und störungsunabhängig scheinen geleitete internetbasierte Angebote eine ähnlich gute Wirksamkeit wie klassische Settings aufzuweisen. Knaevelsrud et al. nennen hierbei Geleitetheit der Intervention, Strukturierung und Dauer, Patientenmerkmale sowie die Synchronität als mögliche Prädiktoren. Insgesamt ist davon auszugehen, dass die therapeutische Beziehung wie im klas- 
sischen Setting auch einen besonders wichtigen Prädikator erfolgreicher therapeutischer Arbeit auch im Videosetting darstellt.

\section{Diskussion}

Die Wirksamkeit von videogestützten und Online-Therapien ist unumstritten und wird vor allem im Falle von geleiteten Therapieangeboten bei verschiedenen Störungsbildern wissenschaftlich nachgewiesen. Indes mangelt es an E-Health-bezogenen Wirksamkeitsstudien zur therapeutischen Behandlung von Autismus-Spektrum-Störungen. Eine videogestützte Therapie mit Personen mit einer vorliegenden ASS und deren Familien scheint trotzdem nicht nur im Kontext der COVID-19-Pandemie indiziert und zugleich operationalisierbar $\mathrm{zu}$ sein. Implikationen reichen von generellen (z. B. bessere Zugänglichkeit/ Erreichbarkeit) über syndromimmanente (z.B. stressfreiere Kommunikation) bis hin $\mathrm{zu}$ altersabhängigen Faktoren (z.B. Mediumvertrautheit bei Jugendlichen). Zentral bleibt jedoch - gerade bei einer Kontaktstörung - die Kontaktqualität und -intensität, sodass auch videogestützte Arbeit als Ergänzung und als Möglichkeit $\mathrm{zu}$ verstehen ist und bloße Online-Angebote ohne Kontakt kontraindiziert erscheinen. Einem kontaktlosen Online-Angebot fehlen schlussendlich das Reale und Fassbare, das Reziproke, die Schwingung und somit der Klang.

\section{》) Therapeutische Allianz/ Beziehung steht im Zentrum}

Zentral ist und bleibt somit neben dem Inhalt der Therapie und den eingesetzten Methoden die therapeutische Allianz/Beziehung, sodass im Mittelpunkt therapeutischer Bemühungen die Frage der Gewährleistung dieser Aspekte steht. Nachdem die inhaltlich-methodische Gestaltung der Therapie im Text behandelt wurde, dürfen die in der Literatur durchaus kritisch beäugten Beziehungsaspekte nicht unberücksichtigt bleiben, wobei inzwischen verschiedene Studien zeigen konnten, dass auch im Videoformat eine gute therapeutische Beziehung aufgebaut werden kann. Neben der Notwendigkeit einer intensiven Kontaktdichte, dem Begreifen des Videoformats als Zusatzangebot und der Empfehlung, erste Kontakte in einem Vis-à-Vis-Format vorzunehmen, sollen weitere autismusspezifische Beziehungsmerkmale wie die autistische therapeutische Haltung in Form eines vorübergehenden - Eintauchens in die autistische Welt oder die Einbeziehung autistischer Objekte Berücksichtigung finden.

Dessen ungeachtet bleiben verschiedene Aspekte erschwert wie etwa die qualitative Diagnostik, gestische und körperliche Hinweissignale sowie insgesamt die Arbeit mit kleinen Kindern. Letztere ist durchaus als besondere Herausforderung und gar Grenze der videogestützten Autismustherapie zu sehen: Während die therapeutische Arbeit per Video als machbar und positiv zu bewerten ist, lässt sich in der Regel die Videoarbeit mit Vorschulkindern kaum realisieren. Hier kann die Videotherapie als kurze Kontaktmöglichkeit im Sinne der therapeutischen Kontinuität verstanden werden, etwa wenn die Vis-à-Vis-Kontakte z. B. aufgrund eines langen Urlaubs oder stationären Aufenthalts des Kindes ausbleiben. Mit Einschränkungen und abhängig vom Symptomgepräge und vom Inhalt lassen sich ebenfalls therapeutische Videositzungen abhalten, wenn ein Elternteil neben dem Kind sitzt und die - vorübergehende - Funktion eines CoTherapeuten einnimmt. Während Therapiepausen aus den genannten oder anderen Gründen kann die Unterstützung und Anleitung der Eltern per Video indiziert sein, etwa um mit bestimmten Krisen besser umgehen zu können, eine Entlastung zu erfahren und dabei unterstützt zu werden, bestimmte Strukturen einzuführen.

\section{Fazit für die Praxis}

- Videogestützte Therapiesitzungen in der Autismustherapie stellen eine Erfolg versprechende Erweiterung der Vis-a-Vis-Arbeit dar und können über die durch die COVID-19-Pandemie bedingte Situation hinaus indiziert sein.

- Wenngleich sich bestimmte Medien wie das klassische Spiel im Videoformat nur mit Einschränkungen realisieren lassen, liegt eine Vielzahl von geeigneten Interventionen für die therapeutische Arbeit mit Kindern, Jugendlichen und Erwachsenen mit Autismus-Spektrum-Störungen (ASS) vor.

- Zwar bedarf es hinsichtlich der Wirksamkeit des Videoformats in der Autismustherapie einer gezielten Erforschung, die Ergebnisse der Forschung in diversen anderen therapeutischen Kontexten zeigen aber positive und somit ermutigende Ergebnisse, sodass auch in der Autismustherapie von einer vergleichbaren Wirksamkeit wie in der klassischen Therapie auszugehen ist.

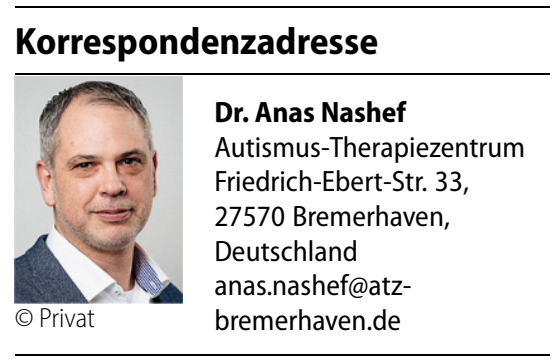

Einhaltung ethischer Richtlinien

Interessenkonflikt. A. Nashef gibt an, dass kein Interessenkonflikt besteht.

Für diesen Beitrag wurden von den Autoren keine Studien an Menschen oder Tieren durchgeführt. Für die aufgeführten Studien gelten die jeweils dort angegebenen ethischen Richtlinien.

Hinweis des Verlags. Der Verlag bleibt in Hinblick auf geografische Zuordnungen und Gebietsbezeichnungen in veröffentlichten Karten und Institutsadressen neutral. 